\title{
Downregulated expression of TSHR is associated with distant metastasis in thyroid cancer
}

\author{
TIANRUN LIU ${ }^{1 *}$, QIANQIAN MEN ${ }^{2 *}, \mathrm{XUAN} \mathrm{SU}^{3}$, WEICHAO CHEN $^{3}$, LAN ZOU $^{3}$, QIULI LI $^{3}$,

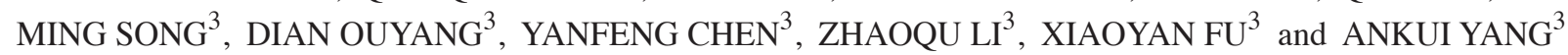 \\ ${ }^{1}$ Department of Otorhinolaryngology Head and Neck Surgery; ${ }^{2}$ Graceland Medical Center, \\ The Sixth Affiliated Hospital of Sun Yat-sen University, Guangzhou, Guangdong 510655; \\ ${ }^{3}$ Department of Head and Neck Surgery, State Key Laboratory of Oncology in South China, \\ Sun Yat-sen University Cancer Center, Guangzhou, Guangdong 510060, P.R. China
}

Received April 15, 2016; Accepted February 13, 2017

DOI: $10.3892 / \mathrm{ol} .2017 .7122$

\begin{abstract}
In differentiated thyroid cancer (DTC), the association between thyroid-stimulating hormone receptor (TSHR) and metastasis, and the underlying molecular mechanisms remain unclear. The role of TSHR in the epithelial-mesenchymal transition (EMT) has not yet been reported, to the best of our knowledge. In the present study, the role of TSHR in the distant metastasis of DTC was investigated. TSHR was significantly downregulated in well-differentiated thyroid cancer cells and tissues, and a lack of TSHR promoted thyroid cancer cell invasion and metastasis by inhibiting the EMT of thyroid cancer cells. In addition, the prognostic value of TSHR in thyroid cancer was analyzed. Immunohistochemical analysis of 172 DTC tissues revealed that a lack of expression of TSHR was associated with distant metastasis and a poor survival rate. Multivariate analyses demonstrated that TSHR expression was a significant prognostic factor for distant metastasis and survival time. The results from the present study demonstrated that TSHR inhibits metastasis through regulating EMT in vitro, and that a lack of expression of TSHR is a significant independent factor affecting distant metastasis and poor prognosis in DTC.
\end{abstract}

\section{Introduction}

Thyroid cancer, which primarily arises from thyroid follicular epithelial cells, is the most common and deadly endocrine malignancy. Well-differentiated thyroid carcinoma (DTC), including papillary thyroid cancer (PTC) and follicular thyroid

Correspondence to: Professor Ankui Yang, Department of Head and Neck Surgery, State Key Laboratory of Oncology in South China, Sun Yat-sen University Cancer Center, 651 Dongfeng Road East, Guangzhou, Guangdong 510060, P.R. China

E-mail: yangak@sysucc.org.cn

\section{${ }^{*}$ Contributed equally}

Key words: differentiated thyroid cancer, TSHR, metastasis, prognosis cancer (FTC), comprise $80 \%$ of all cases of thyroid cancer and frequently have a favorable prognosis; however, $20 \%$ of these patients develop distant metastases or recurrences and eventually succumb to the disease $(1,2)$. Therefore, further studies are required to identify novel prognostic factors for DTC. Once thyroid cancer metastasizes to distant sites and is resistant to radioactive iodine therapy, the expected survival time declines rapidly (3); however, the underlying molecular mechanisms of thyroid cancer metastasis remain unclear.

Through activation by thyroid-stimulating hormone (TSH), the TSH receptor (TSHR) serves a fundamental role in the regulation of thyroid cell proliferation, differentiation and function, in addition to the development of the thyroid gland (4). It has been demonstrated that TSHR signaling was required for thyroid carcinogenesis in a mouse model (5). Additionally, TSH-TSHR signaling has a dichotomous role in the development of thyroid cancer; it can also suppress malignant transformation of thyroid cells and therefore suppress the occurrence of thyroid cancer $(5,6)$. However, the majority of these previous studies investigated the underlying molecular mechanisms of thyroid cancer growth, and the role of TSHR in the prognosis, migration, invasion and metastasis of DTC remains unclear. Epithelial-mesenchymal transition (EMT), a phenotypic plasticity conversion, which is essential in organ morphogenesis, development and tissue remodeling, is typically detected in neoplasias and during cancer progression $(7,8)$. Additionally, EMT is associated with tumor metastasis, and it is thought that EMT endows invasive and stem cell-like features that allow tumor cells to disseminate $(9,10)$.

In the present study, the prognostic value of TSHR for patients with thyroid cancer, and its role in the migration and invasion of thyroid cancer were investigated. Low expression of TSHR was identified to be associated with a high distant metastasis rate and poor prognosis in patients with DTC, and TSHR inhibited thyroid cancer cell migration and invasion.

\section{Materials and methods}

Cell culture. The primary normal human thyroid follicular epithelial cell lines N1 and N2, which were isolated from 
primary thyroid tissues of surgical resection of 2 healthy individuals between August 2008 and April 2014. Written informed consent was provided by all patients. The well-differentiated PTC cell lines B-CPAP, PTC-1113A and PTC-uc3, and the poorly-differentiated thyroid carcinoma cell lines FRO and WRO were cultured in RPMI-1640 medium (Thermo Fisher Scientific, Inc., Waltham, MA, USA) supplemented with $10 \%$ fetal bovine serum (FBS; Gibco; Thermo Fisher Scientific, Inc.), $0.1 \% \mathrm{mM}$ non-essential amino acids, $1 \mathrm{mM}$ sodium pyruvate and $1 \%$ penicillin-streptomycin in a humidified incubator with $5 \% \mathrm{CO}_{2}$ at $37^{\circ} \mathrm{C}$. The PTC cells were obtained from the American Type Culture Collection (Manassas, VA, USA).

Patient information and tissue specimens. A total of 172 formalin-fixed paraffin-embedded thyroid cancer samples were collected at Sun Yat-Sen University Cancer Center (Guangzhou, China) between January 1990 and December 2003. The follow-up time ranged between 120 and 183 months. The clinical classification was performed according to The Chinese 1992 staging system (11).

All the cases included in the present study were histopathologically and clinically diagnosed, and then verified. Written informed consent was obtained from all patients and the present study was approved by the Ethics Committee of Sun Yat-Sen University Cancer Center and sample collection was performed in accordance with the policies of the National Research Ethics Committee of China. Clinical information is summarized in Table I.

$3 D$ morphogenesis assay. Plates (24-well) were coated with Growth Factor Reduced Matrigel (BD Biosciences, Franklin Lakes, NJ, USA) and covered with the RPMI plus $10 \%$ FBS growth medium supplemented with 2\% Matrigel. Cells were trypsinized and then seeded at a density of $1 \times 10^{4}$ cells/well, cultured for 3-4 days. Images were captured with a phase-contrast microscope at 2-day intervals for 2-3 weeks.

Reverse transcription-quantitative polymerase chain reaction $(R T-q P C R)$. Total RNA was isolated using TRIzol reagent (Thermo Fisher Scientific, Inc.), and cDNA was produced using PrimeScript RT reagent kit with gDNA Eraser from $1 \mu \mathrm{g}$ cDNA (Takara Bio, Inc., Otsu, Japan). RT-qPCR was performed using SYBR-Green PCR Master Mix (Takara Bio, Inc.) on a 7500 Fast Real time PCR system (Applied Biosystems; Thermo Fisher Scientific, Inc.). For the PCR reaction, the thermocycler condition was as follows: $50^{\circ} \mathrm{C}$ for $2 \mathrm{~min}$, and then $95^{\circ} \mathrm{C}$ for $10 \mathrm{~min}$, followed by 40 cycles of $95^{\circ} \mathrm{C}$ for $15 \mathrm{sec}$ and $60^{\circ} \mathrm{C}$ for $1 \mathrm{~min}$. $\beta$-actin was used as an endogenous control for gene normalization. Expression data was calculated as $2^{-\Delta \Delta \mathrm{Cq}}(12)$.

Western blotting. Cells were lyzed with radioimmunoprecipitation buffer (Beyotime Institute of Biotechnology, Haimen, China) (2) and the protein concentration was determined using a BCA Protein Assay kit (Pierce; Thermo Fisher Scientific, Inc.) (2). Western blotting was performed using standard methods as described previously (2). Briefly, $40 \mathrm{ug}$ protein were applied to $12 \%$ polyacrylamide SDS gels, separated by SDS-PAGE and transferred onto polyvinylidene fluoride
(PVDF) membranes (GE Healthcare Life Sciences, Chalfont, UK). The membranes were blocked with 5\% no-fat milk at room temperature for $1 \mathrm{~h}$, and incubated with anti-TSHR (dilution, 1:1,000; cat. no. ab27974; Abcam, Cambridge, UK), anti-Vimentin (dilution, 1:1,000; cat. no. 5741; Cell Signaling Technology, Inc., Danvers, MA, USA), anti-N-Cadherin (dilution, 1:1,000; cat. no. 13116; Cell Signaling Technology, Inc.) or anti- $\beta$-actin (dilution, 1:5,000; cat. no. A5441; Sigma-Aldrich) primary antibodies at $4^{\circ} \mathrm{C}$ overnight. Subsequently, the secondary antibodies for Anti-mouse IgG HRP-linked Antibody (dilution, 1:5,000; cat. no. 7056; Cell Signaling Technology, Inc.) and Anti-rabbit IgG, HRP-linked Antibody (dilution, 1:5,000; cat. no. 7074; Cell Signaling Technology, Inc.) were used to incubated with the membranes at room temperature for $2 \mathrm{~h}$. Then an enhanced chemiluminescence Amersham ECL Primer kit (GE Healthcare Life Sciences) was used to develop the blots according to the manufacturer's protocol. $\beta$-actin was used as the loading control.

Wound healing and invasion assays. A total of $1 \times 10^{5}$ cells were seeded and cultured to confluence in 6-well plate, streaks were created in the monolayer with a $200 \mu 1$ pipette tip, migration progression was observed and photographed at indicated time points. For invasion assays, $1 \times 10^{5}$ cells were placed on Matrigel-coated 24-well Boyden chambers (Corning Incorporated, NY, USA). Following $24 \mathrm{~h}$, the non-invading cells were gently removed with a soft cotton swab. The cells that invaded to the bottom chamber were fixed with methanol and glacial acetic acid $(3: 1)$ for $30 \mathrm{~min}$, and stained with $0.1 \%$ crystal violet. Images for the scratch wound and invasion assays were captured using a phase-contrast microscope.

RNA interference. A short interfering (si)RNA directed against TSHR (cat. no. stB0005212A-1-5) was synthesized by Guangzhou RiboBio Co., Ltd. (Guangzhou, China) and the control siRNA (cat. no. siN0581522147-1-5) was purchased from RiboBio. The siRNA transfection was performed in 6-well plates using Lipofectamine ${ }^{\circledR} 2000$ (Invitrogen; Thermo Fisher Scientific, Inc.) according to the manufacturer's protocol.

Immunofluorescence. Cells were seeded onto coverslips 1 day prior to fixation. For immunofluorescence analysis, the cells were probed with the antibodies against N-Cadherin and Vimentin that were also used in the western blot overnight at $4^{\circ} \mathrm{C}$. Following several washes, Alexa Fluor ${ }^{\circledR}$ 594-conjugated secondary antibodies (1:500, cat. no. Z25007; Invitrogen; Thermo Fisher Scientific, Inc.) were used for staining at room temperature for $1 \mathrm{~h}$. All cells were counterstained with DAPI and imaged by confocal laser-scanning microscopy (LSM710; Zeiss GmbH, Jena, Germany).

Immunohistochemistical staining and analysis. Immunohistochemistry (IHC) was performed according to previous methods (5): $4 \mu \mathrm{m}$ paraffin-embedded thyroid cancer tissue sections using monoclonal antibodies directed against TSHR (1:100, cat. no. ab219322; Abcam) at room temperature for $1 \mathrm{~h}$. The secondary antibody Goat Anti-Mouse IgG H\&L (HRP) (cat. no. ab205719; Abcam) was incubated at room 
Table I. Association between TSHR expression and the clinicopathological features of 172 patients with DTC.

TSHR expression, no. of patients

Clinicopathological feature

Negative Positive

$\chi^{2}$

P-value

Tissue

Normal thyroid tissue

15

79

DTC tissue

Gender

Male

Female

Age, years

$<45$

$\geq 45$

$$
32
$$

Pathological type

Papillary

Follicular

58

21

54

25

26

53

$\mathrm{N}+$

Distant metastasis

M0

M1

Recurrent laryngeal nerve invasion

Yes

No

68

11

5

74

13

66

18

61

Extracapsular invasion

Yes

Trachea invasion

Yes

\section{6}

93

6.856

0.009

31

62

0.947

0.331

56

37

1.651

0.199

83

10

0.401

0.527

70

23

0.077

0.782

74

19

4.423

0.219

38

55

1.155

0.282

89

4

4.969

0.026

8

85

0.316

0.574

19

74

0.446

0.504

15

78

1.22

0.269

TSHR, thyroid-stimulating hormone receptor; DTC, differentiated thyroid cancer; T, tumor; N, node.

temperature for $15 \mathrm{~min}$. Tissue sections were observed under an AX10-Imager A1 (Zeiss GmbH), and all images were captured using AxioVision microscopy software (version 4.7; Zeiss). The score was evaluated by estimating the percentage and intensity of tumor cell staining. The scores of positively stained tumor cells were graded as: 0 (no positive tumor), 1 $(<10 \%), 2(10-50 \%)$ and $3(>50 \%)$. The intensity of tumor cell staining was determined as: 0 (no staining), 1 (light yellow), 2 (yellow brown), 3 (brown). The staining index was calculated as the product of staining intensity $x$ percentage of positive tumor cells, resulting in scores of $0,1,2,3,4,6$ and 9 .
Statistical analysis. All statistical analyses were performed using SPSS software (version 19.0; IBM SPSS, Armonk, NY, USA). The $\chi^{2}$ test was used to analyze the association between TSHR expression and the clinicopathologic characteristics of patients. Logistic regression was used to analyze the factors associated with distant metastasis in patients with DTC. Survival curves were plotted using the Kaplan-Meier estimator method and compared using the log-rank test. Survival data were evaluated using multivariate Cox regression analyses. $\mathrm{P}<0.05$ was considered to indicate a statistically significant difference. 

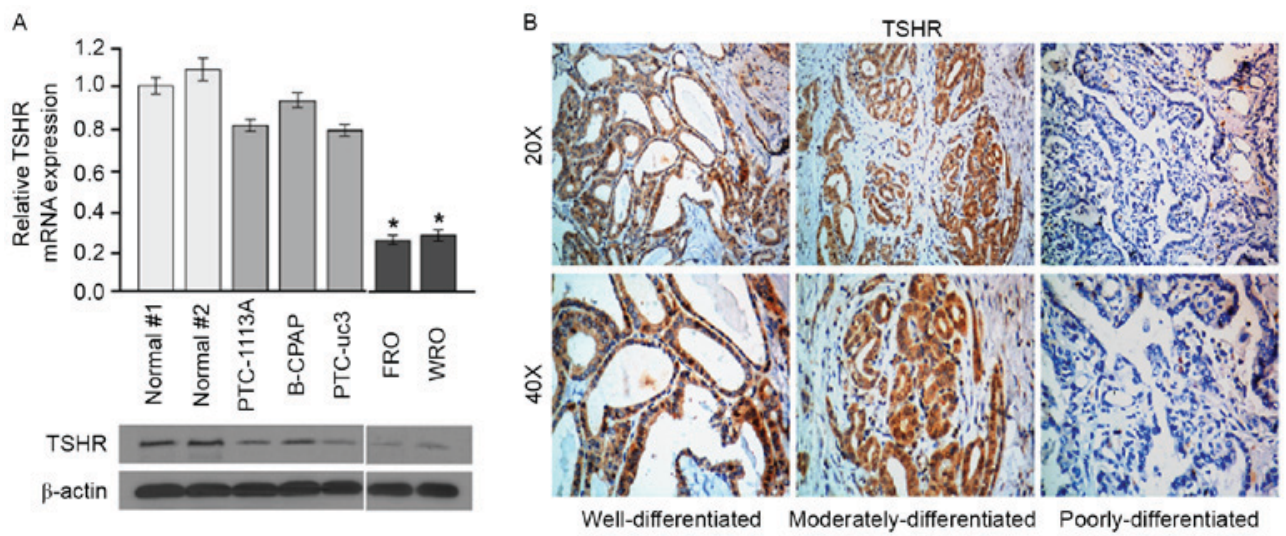

Figure 1. Downregulation of TSHR in differentiated thyroid cancer cell lines and tissues. (A) Reverse transcription-quantitative polymerase chain reaction and western blotting analysis of TSHR expression in normal thyroid tissues and thyroid cancer cell lines, including B-CPAP, PTC-113A, PTC-uc3, FRO and WRO. (B) Representative images of TSHR protein expression examined by immunohistochemical analysis. * $\mathrm{P}<0.05$ in poorly differentiated thyroid cancer cells vs. well-differentiated thyroid cancer cells. TSHR, thyroid-stimulating hormone receptor.

\section{Results}

TSHR is downregulated in poorly-differentiated thyroid cancer cell lines and tissues. To investigate the role of TSHR in thyroid cancer, TSHR levels in normal thyroid cells and thyroid cancer cells with different differentiation stages were determined using western blotting and RT-qPCR analysis. All differentiated and poorly-differentiated thyroid cancer cell lines studied expressed TSHR mRNA and protein, and expression levels of TSHR mRNA were significantly higher in the well-differentiated cell lines (PTC-113A, B-CPAP and PTC-uc3) compared with the poorly-differentiated cell lines (FRO and WRO) (Fig. 1A). To confirm this result, the expression of TSHR in thyroid cancer tissues was determined using immunohistochemical analysis. The well-differentiated thyroid cancer tissues exhibited higher TSHR expression compared with moderately- and poorly-differentiated thyroid cancer tissues (Fig. 1B). These results demonstrate that TSHR is downregulated in poorly differentiated DTC cell lines and clinical tissues.

Downregulation of TSHR promotes thyroid cancer cell invasion and metastasis. The importance of TSHR in thyroid cancer cell metastasis in the well-differentiated cancer cell line B-CPAP was investigated. The 3D culture model revealed that the invasive and metastatic ability of cells was markedly increased when TSHR expression was silenced in B-CPAP cell lines compared with the control siRNA group (Fig. 2A). In addition, similar results were obtained from the wound healing and invasion assays (Fig. 2B and C, respectively). These results indicate that the downregulation of TSHR promotes the invasion and metastasis of DTC. EMT is associated with the invasion and metastasis of tumors, so immunofluorescence and western blotting was performed to examine the expression of markers of EMT in thyroid cancer cells. In the well-differentiated thyroid cancer cell line B-CPAP, high expression of TSHR inhibited the expression of two classical mesenchymal cell markers, $\mathrm{N}$-cadherin and vimentin (Fig. 2D and E). When TSHR was silenced, the expression of $\mathrm{N}$-cadherin and vimentin was recovered (Fig. 2D and E). These results suggest that TSHR suppresses thyroid cancer cell invasion and metastasis by inhibiting EMT.

Low expression of TSHR is associated with a high distant metastasis rate in patients with DTC. To further investigate the role of TSHR in thyroid cancer progression, immunohistochemical staining of TSHR levels was statistically analyzed to determine their association with the clinical features of patients with DTC (Table I). TSH expression was detected in 93/172 DTC cases (54.1\%). Distant metastasis occurred in $15 / 172$ cases $(8.72 \%)$, including lung metastasis in 14 cases and liver metastasis in 1 case. The distant metastasis rates in the TSHR-negative and -positive groups were 13.92 and $4.30 \%$, respectively $(\mathrm{P}=0.026)$. Logistic regression was performed to analyze the factors associated with distant metastasis in patients with DTC (Table II). The results revealed that TSHR expression was a significant independent factor affecting distant metastasis in patients with DTC $(\mathrm{P}=0.035)$. Distant metastasis status was also associated with age, pathological type, T stage, and primary treatment; however, it was not associated with $\mathrm{N}$ stage, recurrent laryngeal nerve invasion, extracapsular invasion or trachea invasion (data not shown).

TSHR expression is an independent prognostic factor for patients with DTC. As age is the most important independent prognostic factor for DTC (13), the present study demonstrated that the 10-year overall survival (OS) rates were 98.2 and $61.2 \%$ in the $<45$ and $\geq 45$-year-old groups, respectively $\left(\mathrm{x}^{2}=43.335 ; \mathrm{P}<0.001\right)$. Therefore, a stratified prognostic analysis was performed according to age, and patients who were $\geq 45$ years old were selected for survival analysis. Among patients with DTC who were $>45$ years old, the survival time was significantly different between the patients with low and high TSHR expression as measured by IHC scores $\left(x^{2}=4.863\right.$; $\mathrm{P}=0.027$; data not shown), and the TSHR-positive group had a 10 -year OS of $73.8 \%$, whereas the OS in the TSHR-negative group was only $41.0 \%$ (Fig. 3). In addition, a multivariate analysis demonstrated that TSHR expression, pathological stage, distant metastasis, $\mathrm{N}$ stage and primary treatment were significant independent prognostic factors in patients with DTC (all P<0.05; Table III). 
A

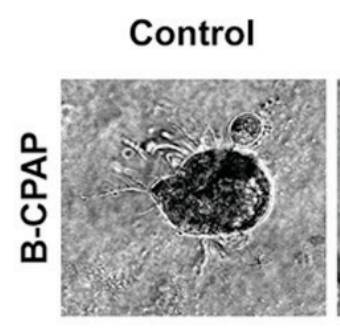

C

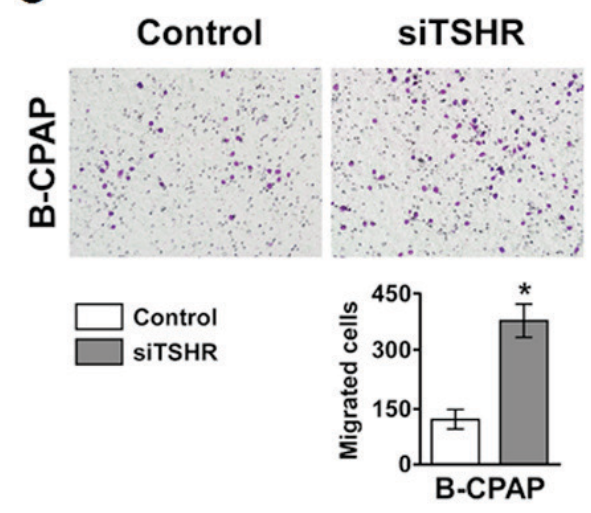

D

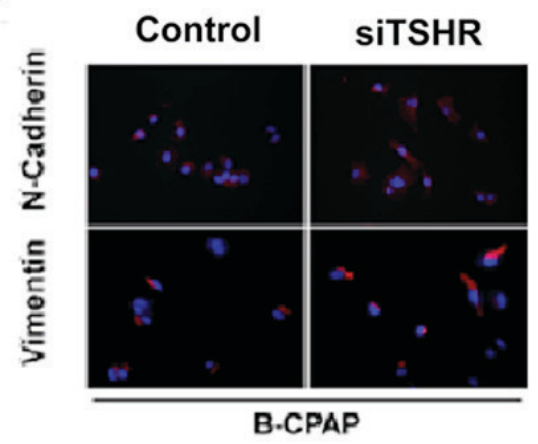

B

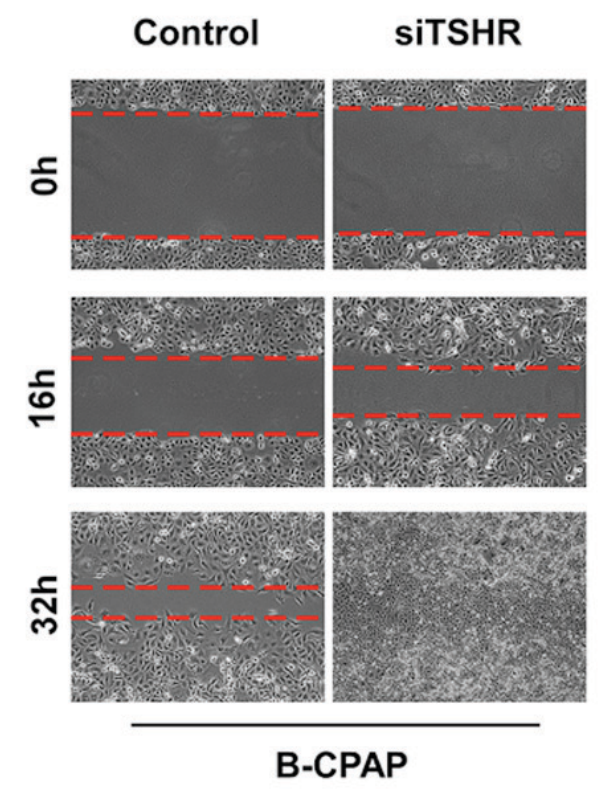

$\mathbf{E}$

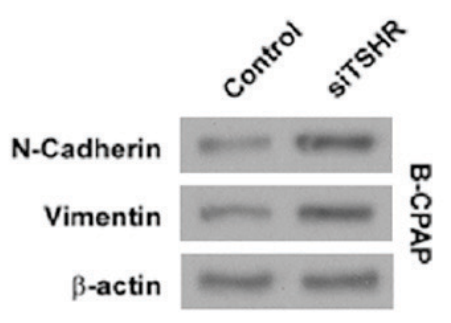

Figure 2. Downregulation of TSHR promotes thyroid cancer cell invasion, and TSHR suppresses EMT in thyroid cancer. (A) Representative micrographs of the indicated cells following an 8-day culture in three-dimensional spheroid invasion assays. Magnification, x400. (B) The mobility of cells with different treatments was measured by testing the rate of wound closure at different time points. Magnification, x200. (C) The invasive properties of thyroid cancer cells with different treatments were analyzed by an invasion assay using a Matrigel-coated Boyden chamber. Migrated cells were plotted as the average number of cells/field of view from 3 different experiments. Magnification, x200. Error bars represent the standard error of the mean (n=3). (D) Immunofluorescence staining of $\mathrm{N}$-cadherin and vimentin expression in differently treated thyroid cancer cell lines. (E) Western blotting analysis of $\mathrm{N}$-cadherin and vimentin expression in different treatments of thyroid cancer cell lines. " $\mathrm{P}<0.05$ vs. the control group. TSHR, thyroid-stimulating hormone receptor; EMT, epithelial-mesenchymal transition.

\section{Discussion}

Thyroid cancer is a major cause of mortality and morbidity. A number of studies have been performed to elucidate the underlying molecular mechanisms that regulate thyroid cancer proliferation and progression $(1,8)$. However, the underlying molecular mechanisms of the distant metastasis of thyroid cancer remain unclear $(14,15)$. It has been demonstrated that several signaling pathways, including the TSHR signaling pathway, contribute to the development of thyroid cancer (16); however, the association between the TSHR signaling pathway and metastasis has not yet been reported, to the best of our knowledge. The present study demonstrated that TSHR inhibits the invasion and metastasis of the well-differentiated cancer cell line B-CPAP in vitro.
TSHR is typically considered to be an oncogene in thyroid cell carcinogenesis and the expression of TSHR has been correlated with poor patient outcomes (17); however, another study reported that aberrant methylation of the TSHR gene in epithelial thyroid cancers leads to TSHR expression silencing and malignant epithelial thyroid tumors $(5,17)$. The results from the present study suggest that the expression of TSHR can suppress the invasive and metastatic abilities of thyroid cancer cells in vitro. Furthermore, analysis of the clinicopathologic characteristics of patients with DTC indicated that the expression of TSHR is associated with good prognosis.

Metastasis is an essential feature of tumors and accounts for the majority of cancer-associated mortalities in humans (18). EMT is a key morphological change of cells that can facilitate the dissemination of cells from the original organ to a distant 
Table II. Logistic regression results of the clinicopathological factors associated with distant metastasis in patients with DTC.

\begin{tabular}{lccccrrr}
\hline & & & & & & \multicolumn{2}{c}{$\operatorname{Exp}(\mathrm{B}) 95 \%$ CI } \\
\cline { 4 - 8 } Clinicopathological factor & B & SE & Wals & P-value & Exp (B) & Lower & Upper \\
\hline Age & 1.522 & 0.771 & 3.898 & 0.048 & 4.583 & 1.011 & 20.771 \\
TSHR expression & -1.469 & 0.696 & 4.450 & 0.035 & 0.230 & 0.059 & 0.901 \\
Pathological type & 3.044 & 0.877 & 12.051 & 0.001 & 20.987 & 3.763 & 117.035 \\
T stage & 0.615 & 0.314 & 3.830 & 0.050 & 1.849 & 0.999 & 3.423 \\
N stage & 1.408 & 0.844 & 2.780 & 0.095 & 4.086 & 0.781 & 21.374 \\
Primary treatment & 1.372 & 0.667 & 4.236 & 0.040 & 3.943 & 1.068 & 14.560 \\
\hline
\end{tabular}

TSHR, thyroid-stimulating hormone receptor; CI, confidence interval; T, tumor; N, node; B, interpretation coefficient; SE, standard error; Wals, Wals statistics.

Table III. Multivariate analysis results of the clinicopathological factors affecting the prognosis of patients with DTC that were $\geq 45$ years old.

\begin{tabular}{lrcccccr}
\hline & & & & & & \multicolumn{2}{c}{ Exp (B) 95\% CI } \\
\cline { 3 - 7 } Clinicopathological factor & B & SE & Wald & P-value & Exp (B) & Lower & Upper \\
\hline TSHR expression & -1.107 & 0.467 & 5.623 & 0.018 & 0.331 & 0.132 & 0.825 \\
N stage & 2.094 & 0.799 & 6.865 & 0.009 & 8.117 & 1.695 & 38.877 \\
Pathological stage & -1.506 & 0.845 & 3.171 & 0.075 & 0.222 & 0.042 & 1.164 \\
Distant metastasis & 1.957 & 0.468 & 17.450 & 0.000 & 7.076 & 2.825 & 17.720 \\
Primary treatment & 0.991 & 0.440 & 5.070 & 0.024 & 2.695 & 1.137 & 6.386 \\
\hline
\end{tabular}

DTC, differentiated thyroid cancer; TSHR, thyroid-stimulating hormone receptor; T, tumor; N, node; B, interpretation coefficient; SE, standard error; Wals, Wals statistics.

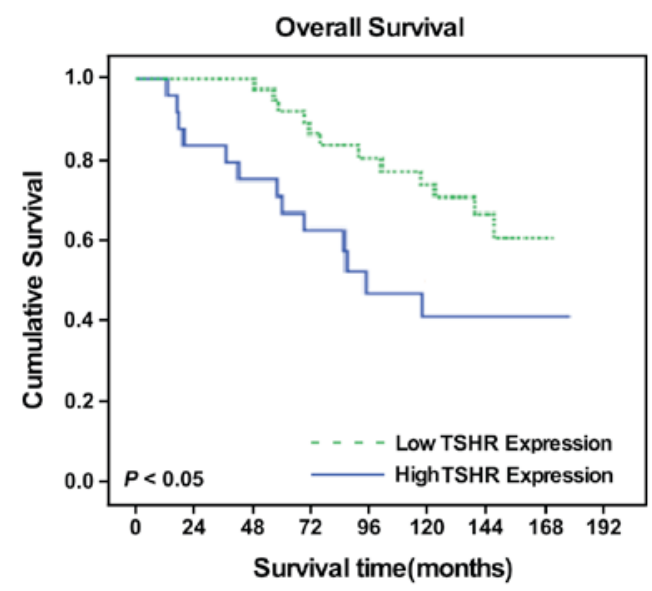

Figure 3. The prognostic value of TSHR in patients with differentiated thyroid cancer that were $\geq 45$ years old. The overall survival time of patients with thyroid cancer with low TSHR vs. high TSHR-expressing tumors was plotted using the Kaplan-Meier estimator method. TSHR, thyroid-stimulating hormone receptor.

site. EMT is a common event, which is frequently observed during cancer development and progression $(8,9)$. The data from the present study suggests that TSHR expression can suppress thyroid cancer cell metastasis by inhibiting thyroid cancer cell EMT in vitro. This indicates that TSHR expression could be a biomarker and independent prognostic factor for thyroid cancer in the clinic. However, it is not clear how TSHR suppresses EMT. Previous studies have demonstrated that microRNA (miRNA/miR) serves an important role in EMT; for example, miR-146b-5p induces EMT through the regulation of Wnt/ $\beta$-catenin signaling $(19,20)$ and the miR-200 family inhibits polycomb complex protein BMI1, and zinc finger E-box-binding homeobox 1 and 2, to suppress EMT (21-23). Further studies are required to demonstrate whether TSHR regulates EMT through miRNAs.

Data from the present study demonstrated that a lack of expression of TSHR was associated with distant metastasis and a poor survival rate in patients with DTC. The distant metastasis rate in the TSHR negative group was significantly higher compared with that of the TSHR positive group (13.92 vs. $4.30 \%$, respectively) and the lung was the most common metastasis site. TSHR expression was also a significant independent factor affecting distant metastasis in patients with DTC. N stage was not associated with distant metastasis; however, a study from Jeon et al (24) demonstrated that the location of associated lymph node (LN) metastasis categories is more useful than the amount of associated LNs categories 
for estimating the risk of distant metastasis in PTC. The use of molecular biomarkers, including TSHR, combined with clinicopathological characteristics may aid in the accurate prediction of the incidence of distant metastases in patients with thyroid cancer.

Endocrine therapy has been used in clinical practice for $>30$ years. Theoretically, the downregulation of TSH through suppression of pituitary TSH feedback by oral thyroxine may inhibit the proliferation of PTC cells, which may reduce the recurrence rate of the tumor; however, the exact treatment effect is not clear and there is a lack of strong clinical evidence (25-27). The results from the current study regarding the role of TSHR on distant metastases in DTC may provide some theoretical basis for the application of endocrine treatment options in DTC. However, prospective clinical trials are required to evaluate whether TSH endocrinal treatment can significantly improve the survival rate of patients by inhibiting distant metastasis.

In conclusion, the present study identified that a lack of expression of TSHR was associated with distant metastases and poor survival time in patients with DTC. In addition, the expression of TSHR was revealed to reduce thyroid cancer cell metastatic capability by inhibiting EMT. Further clinical studies are required to elucidate the diagnostic and therapeutic implications of the role or TSHR in thyroid tumors, and prospective clinical trials are required to evaluate whether TSH endocrinal treatment can significantly decrease the incidence of distant metastasis in these patients.

\section{Acknowledgements}

The current study was supported by the National Science Foundation of China (grant nos. 81272955 and 81302368) and the Guangdong Province Natural Science Foundation (grant nos. 2014A020212100, S2011010003997 and 2016A020215082).

\section{References}

1. Nikiforov YE and Nikiforova MN: Molecular genetics and diagnosis of thyroid cancer. Nat Rev Endocrinol 7: 569-580, 2011.

2. Cras A, Politis B, Balitrand N, Darsin-Bettinger D, Boelle PY, Cassinat $\mathrm{B}$, Toubert $\mathrm{ME}$ and Chomienne $\mathrm{C}$ : Bexarotene via $\mathrm{CBP} / \mathrm{p} 300$ induces suppression of NF- $\mathrm{BB}$-dependent cell growth and invasion in thyroid cancer. Clin Cancer Res 18: 442-453, 2012.

3. Shoup M, Stojadinovic A, Nissan A, Ghossein RA, Freedman S, Brennan MF, Shah JP and Shaha AR: Prognostic indicators of outcomes in patients with distant metastases from differentiated thyroid carcinoma. J Am Coll Surg 197: 191-197, 2003.

4. Kimura T, Van Keymeulen A, Golstein J, Fusco A, Dumont JE and Roger PP: Regulation of thyroid cell proliferation by TSH and other factors: A critical evaluation of in vitro models. Endocr Rev 22: 631-656, 2001.

5. Lu C, Zhao L, Ying H, Willingham MC and Cheng SY: Growth activation alone is not sufficient to cause metastatic thyroid cancer in a mouse model of follicular thyroid carcinoma. Endocrinology 151: 1929-1939, 2010.

6. Davies T, Marians R and Latif R: The TSH receptor reveals itself. J Clin Invest 110: 161-164, 2002.

7. Cannito S, Novo E, di Bonzo LV, Busletta C, Colombatto S and Parola M: Epithelial-mesenchymal transition: From molecular mechanisms, redox regulation to implications in human health and disease. Antioxid Redox Signal 12: 1383-1430, 2010.
8. Lamouille S, Xu J and Derynck R: Molecular mechanisms of epithelial-mesenchymal transition. Nat Rev Mol Cell Biol 15: 178-196, 2014.

9. Thiery JP: Epithelial-mesenchymal transitions in tumour progression. Nat Rev Cancer 2: 442-454, 2002.

10. Rhim AD, Mirek ET, Aiello NM, Maitra A, Bailey JM, McAllister F, Reichert M, Beatty GL, Rustgi AK, Vonderheide RH, et al: EMT and dissemination precede pancreatic tumor formation. Cell 148: 349-361, 2012.

11. Liao WT, Song LB, Zhang HZ, Zhang X, Zhang L, Liu WL, Feng Y, Guo BH, Mai HQ, Cao SM, et al: Centromere protein $\mathrm{H}$ is a novel prognostic marker for nasopharyngeal carcinoma progression and overall patient survival. Clin Cancer Res 13: 508-514, 2007.

12. Livak KJ and Schmittgen TD: Analysis of relative gene expression data using real-time quantitative PCR and the 2(-Delta Delta C(T)) Method. Methods 25: 402-408, 2001.

13. Krook KA, Fedewa SA and Chen AY: Prognostic indicators in well-differentiated thyroid carcinoma when controlling for stage and treatment. Laryngoscope 125: 1021-1027, 2015.

14. Gandolfi G, de Biase D, Sancisi V, Ragazzi M, Acquaviva G, Pession A, Piana S, Tallini G and Ciarrocchi A: Deep sequencing of KIT, MET, PIK3CA, and PTEN hotspots in papillary thyroid carcinomas with distant metastases. Endocr Relat Cancer 21: L23-L26, 2014.

15. Gandolfi G, Ragazzi M, Frasoldati A, Piana S, Ciarrocchi A and Sancisi V: TERT promoter mutations are associated with distant metastases in papillary thyroid carcinoma. Eur J Endocrinol 172: 403-413, 2015.

16. Cancer Genome Atlas Research Network: Integrated genomic characterization of papillary thyroid carcinoma. Cell 159: 676-690, 2014.

17. Xing M, Usadel H, Cohen Y, Tokumaru Y, Guo Z, Westra WB, Tong BC, Tallini G, Udelsman R, Califano JA, et al: Methylation of the thyroid-stimulating hormone receptor gene in epithelial thyroid tumors: A marker of malignancy and a cause of gene silencing. Cancer Res 63: 2316-2321, 2003.

18. Hanahan D and Weinberg RA: Hallmarks of cancer: The next generation. Cell 144: 646-674, 2011.

19. Deng X, Wu B, Xiao K, Kang J, Xie J, Zhang X and Fan Y: MiR-146b-5p promotes metastasis and induces epithelial-mesenchymal transition in thyroid cancer by targeting ZNRF3. Cell Physiol Biochem 35: 71-82, 2015.

20. Hardin H, Guo Z, Shan W, Montemayor-Garcia C, Asioli S, Yu XM, Harrison AD, Chen H and Lloyd RV: The roles of the epithelial-mesenchymal transition marker PRRX1 and miR-146b-5p in papillary thyroid carcinoma progression. Am J Pathol 184: 2342-2354, 2014.

21. Braun J, Hoang-Vu C, Dralle $H$ and Hüttelmaier S: Downregulation of microRNAs directs the EMT and invasive potential of anaplastic thyroid carcinomas. Oncogene 29: 4237-4244, 2010.

22. Zhang Z, Liu ZB, Ren WM, Ye XG and Zhang YY: The miR-200 family regulates the epithelial-mesenchymal transition induced by EGF/EGFR in anaplastic thyroid cancer cells. Int $\mathrm{J}$ Mol Med 30: 856-862, 2012.

23. Feng X, Wang Z, Fillmore R and Xi Y: MiR-200, a new star miRNA in human cancer. Cancer Lett 344: 166-173, 2014.

24. Jeon MJ, Kim TY, Kim WG, Han JM, Jang EK, Choi YM, Song DE, Yoon JH, Chung KW, Hong SJ, et al: Differentiating the location of cervical lymph node metastasis is very useful for estimating the risk of distant metastases in papillary thyroid carcinoma. Clin Endocrinol (Oxf) 81: 593-599, 2014.

25. Middendorp M and Grünwald F: Update on recent developments in the therapy of differentiated thyroid cancer. Semin Nucl Med 40: 145-152, 2010.

26. Cooper DS, Specker B, Ho M, Sperling M, Ladenson PW, Ross DS, Ain KB, Bigos ST, Brierley JD, Haugen BR, et al: Thyrotropin suppression and disease progression in patients with differentiated thyroid cancer: Results from the National Thyroid Cancer Treatment Cooperative Registry. Thyroid 8: 737-744, 1998.

27. Sugitani I and Fujimoto Y: Does postoperative thyrotropin suppression therapy truly decrease recurrence in papillary thyroid carcinoma? A randomized controlled trial. J Clin Endocrinol Metab 95: 4576-4583, 2010 